\title{
Wegen „Spendenzahlungen“ Chefarzt wegen Bestechlichkeit und Betrug verurteilt
}

Der Bundesgerichtshof bestätigt mit Beschluss vom 13.07.2011 (Az.: 1 StR 692/10) eine Entscheidung des Landgerichts Essen, wonach ein Universitätsprofessor und Chefarzt für die Klinik für Allgemein- und Transplantationschirurgie eines Universitätsklinikums wegen Bestechlichkeit, Nötigung, Betrug und Steuerhinterziehung zu einer Freiheitsstrafe verurteilt wurde.

\section{Der Fall}

Die „Spendenzahlungen“"

Der beamtete Chefarzt forderte in 30 Fällen von stationär aufgenommenen Regelleistungspatienten, die keinen Anspruch auf eine wahlärztliche Behandlung durch ihn hatten, sogenannte "Spenden“ in Höhe von $2000 €$ bis $7500 €$ pro Fall. Als Gegenleistung für diese „Spenden“ versprach er, die Patienten persönlich zu behandeln. Die Patienten blieben aber dennoch Regelleistungspatienten. Entsprechend der Abrede mit den Patienten bevorzugte er diese, indem er sie gerade aufgrund der zugesagten oder bereits vor Behandlungsbeginn gezahlten Spenden selbst operierte. Die Gelder wurden von dem Chefarzt mit einer Ausnahme auf ein vom Universitätsklinikum verwaltetes Drittmittelkonto gezahlt, über das er faktisch selbst verfügen konnte. In 3 Fällen setzte der Angeklagte die Patienten auBerdem unter Druck, indem er eine von ihm als erforderlich dargestellte zeitnahe Operation bzw. in einem Fall eine Operation, die angeblich nur er persönlich vornehmen konnte, von einer "Spende“ abhängig machte und veranlasste die Patienten auf diese Weise zur Zahlung. In einem weiteren Fall erklärte der Angeklagte dem Patienten, er werde die Operation selbst durchführen, obwohl ihm bekannt war, dass er wegen eines anderen Termins dazu nicht vollständig in der Lage sein würde. Insgesamt erfolgten Spendenzahlungen in Höhe von $158000 €$ für einen Zeitraum von 2003 bis 2007.

Chefarzt profitiert von Drittmittelkonto Der Angeklagte profitierte von den Geldern auf dem Drittmittelkonto, indem er teilweise seine eigenen Reisekosten damit beglich. Darüber hinaus verbesserten sich seine Forschungs- und Arbeitsbedingungen, indem er Arbeitsmittel beschaffen und Reise- und sonstige Kosten für Fortbildungen der Mitarbeiter finanzieren konnte. Seine Sekretärinnen erhielten entsprechend seiner Zusage monatliche Gehaltszulagen vom Drittmittelkonto.

Nach Auffassung des Gerichts fehlte dem Chefarzt bei seinem Einfordern der Spenden zwar das Bewusstsein, gegen das Verbot des $\S 332$ Abs. 1 StGB (Bestechlichkeit) zu handeln. Er hielt seine Praxis der Spenden-Einwerbung nicht für verbotenes Unrecht, wenn die erhaltenen Gelder einem „guten Zweck“ dienten und bei ihm persönlich nichts „hängen bleibe“. Nach Auffassung des Gerichts hätte der Angeklagte jedoch jedenfalls bei gehöriger Erkundigung zu einer Unrechtseinsicht kommen können. Auch der Einlassung des Chefarztes, die Spenden seien freiwillig und aus Dankbarkeit erfolgt, konnte das Gericht nicht folgen.

Verurteilung wegen Bestechlichkeit

Der Chefarzt hat sich nach Auffassung des Landgerichts Essen (Urteil vom 10.3.2010, A2: 56 Kls 20/08), die nun vollumfänglich vom Bundesgerichtshof bestätigt wurde, einer Bestechlichkeit gemäß $\S 332$ Abs. 1 StGB strafbar gemacht. Der Chefarzt war als Amtsträger tätig. Er hat einen Vorteil zunächst gefordert bzw. sich versprechen lassen und schließlich auch angenommen. Dieser Vorteil wurde für eine Dienstleistung erbracht. Zwar hatte der angeklagte Chefarzt die Möglichkeit, privatärztliche Vereinbarungen mit den Patienten zu treffen. Dies ist jedoch ausdrücklich nicht geschehen, sondern die Patienten blieben Regelleistungspatienten und zahlten lediglich eine „Spende“. Die vom Chefarzt vorgenommene Diensthandlung (Operation) verletzte auch seine Dienstpflichten. Als Chefarzt war der Angeklagte sogenannter Ermessensbeamter. Er konnte als Leiter der Klinik bestimmen, wie sich die konkrete Behandlung eines Patienten im Detail vollzog. Insbesondere konnte er bestimmen, durch wen eine Operation durchgeführt wurde und ob ein Patient auf seiner „Privatstation“ mit seinen Visiten aufgenommen wurde. Als Beamter
Gat er seine Entscheidung jedoch ausschließlich an sachlichen Kriterien auszurichten, Die möglichen Kriterien ergeben sichaus dem Aufgabenbereich des Angeklagten in Forschung, Lehre und Krankenversorgung $_{2}$ sind also beispielsweise wissenschaftliches Interesse, Ausbildung oder besondere medizinische Schwierigkeit. Als Ermessensbeamter handelt der Chefarzt bereits dann pflichtwidrig, wenn er sich bei seiner Entscheidung von dem Vorteil beeinflussen lässt, selbst wenn sie innerhalb seines Ermessensspielraums liegt. In den konkreten Fällen ließ sich nach Auffassung der Richter der Angeklagte bei seiner Entscheidung maßgeblich durch die Spende leiten. Er orientierte sich daher gerade nicht an den Sachkriterien, was zu einer Rechtswidrigkeit der Diensthandlung führt. Ob die Entscheidung inhaltlich zutreffend war, es also beispielsweise aufgrund des Krankheitsbildes der Patienten oder aufgrund anderer Gründe gerechtfertigt war, dass gerade der Chefarzt die Patienten behandelte, spielt keine Rolle.

Über den Eigenvorteil hinaus sah das Gericht in den Zahlungen auch einen Vorteil für die Universität, der die Gelder auf dem Drittmittelkonto rechtlich zugeordnet waren, auch wenn das Universitätsklinikum sie verwaltete.

Abschließend kam das Gericht auch zu dem Ergebnis, dass der Chefarzt keinen Anspruch auf die Spenden hatte.

\section{Zusätzliche Nötigung}

In dem einen Fall, in dem die Bestechlichkeit in Tateinheit mit Nötigung bestraft wurde, ging es um eine Patientin, die wegen einer Krebserkrankung operiert wurde. Bei der Operation stellte sich heraus, dass sie an Metastasen litt. Der behandelnde Chirurg erklärte ihr, dass sie dringend erneut operiert werden müsste. Der angeklagte Chefarzt sei ein geeigneter Spezialist. Daher wurde ein Termin für die Patientin in der Privatsprechstunde des Angeklagten vereinbart. Die Patientin 
suchte sodann den angeklagten Chefarzt auf. In dem Gespräch erklärte er ihr unter anderem, dass die Wartezeit für „Kassenpatienten“ ca. 4-6 Wochen betrage. Er selbst könne die Patientin nicht operieren, weil sie eine Kassenpatientin sei. Er habe aber auf seiner Station gerade ein Bett frei. Daher bestehe die Möglichkeit, dass er die Patientin operiere. Sie müsse dann allerdings eine Spende für Forschungszwecke und Kongresse in Höhe von $5000 €$ leisten. Dann werde sie ganz kurzfristig aufgenommen und von ihm operiert. Sie könne die Wartezeit durch die Spende ausgleichen. Die Familie müsse sich aber schnell entscheiden, noch am selben Tag, „sonst ist das Bett weg“. In einem späteren Telefongespräch mit dem Angeklagten konnte die Summe auf $3500 €$ reduziert werden. Die Zahlung erfolgte nach der Operation.

\section{Gericht bescheinigt inakzeptables}

\section{Verhalten}

Im Vorgehen des Chefarztes sahen die Richter eine Nötigung ( $\$ 240$ Abs. 1 StGB). Eine Nötigung begeht, wer einen Menschen rechtswidrig durch Drohung mit einem empfindlichen Übel zu einer Handlung nötigt. Der Angeklagte erklärte der Patientin, als Kassenpatientin betrage ihre Wartezeit auf eine Operation 4-6 Wochen. Der Erklärungswert dieser Aussage war, dass weder er noch ein ihm unterstehender Arzt der Klinik die Patientin vor Ablauf dieser Frist operieren werde. Der Angeklagte stellte damit das Unterlassen einer zeitnahen Operation durch die Ärzte der Klinik und damit eine Verringerung der Überlebenschancen in Aussicht. Denn der Angeklagte hatte eine Überlebenschance von 80:20 nur bei einer schnellen Operation diagnostiziert, womit gleichzeitig klar dargestellt war, dass jedes Zuwarten die Überlebenschance sinken ließ. Durch diese Drohung ist die Patientin zur Zahlung eines Geldbetrages in Höhe von $3500 €$ genötigt worden. Die Tatsache, dass der Chefarzt die Vergabe einer Behandlungskapazität nicht von einer medizinischen Notwendigkeit abhängig machte, sondern von einer Geldzahlung, sah das Gericht als verwerflich an. Er verkaufte ein Bett und eine freie Operationskapazität an eine schwerkranke Patientin, die nach seiner Darstellung ihrer Überlebenschancen auf eine schnelle Operation angewiesen war. Dieses Verhalten erachtete die Kammer als absolut unerträglich und gesteigert 
sozialwidrig. Die Kammer erlaubt sich an dieser Stelle auch einen deutlichen mahnenden Fingerzeig an die Krankenhauspraxis. Die Richter teilen in dem Urteil mit, dass ihnen bewusst sei, dass es sich auch im öffentlichen Krankenhauswesen eingeschlichen habe, Wahlleistungspatienten unter verschiedenen Gesichtspunkten bevorzugt zu behandeln. Das Universitätsklinikum hätte wegen des abzuführenden Nutzungsentgelts auch ein eigenes wirtschaftliches Interesse daran, dass der Chefarzt besonders viele Wahlleistungspatienten behandelte. Dies habe indes keine Konsequenz für die rechtliche Beurteilung. Für die rechtliche Beurteilung des Handelns des Angeklagten sind nicht die deformierte Praxis, sondern rechtliche Maßstäbe anzulegen. Jedenfalls ist eine zeitlich bevorzugte Behandlung einer Wahlleistungspatientin von den für den Angeklagten maßgeblichen Vorschriften nicht gedeckt. Die Kammer bezweifelt, dass eine derartige Praxis bei schwerkranken Patienten und möglicherweise lebensrettenden Operationen existierte. Jedenfalls sei ein derartiges Verhalten völlig inakzeptabel und rechtsstaatlich nicht tolerabel.

Spende war verkappte Geldleistung Auch der vom Angeklagten angestrebte Zweck sei sozialwidrig. Auch wenn der Zahlungsfluss auf ein Drittmittelkonto für Forschungszwecke noch sozial erwünscht sein möge, gelte dies jedenfalls dann nicht mehr, wenn es sich wie im konkreten Fall um eine Zahlung im Austauschverhältnis handele. Denn der Sache nach handelte es sich hier nicht um eine Spende, sondern um eine verkappte Gegenleistung für eine Operation. Bezüglich der Nötigung gingen die Richter auch von einer vorsätzlichen Tat des Chefarztes aus.

\section{Keine persönliche Leistungserbringung - Betrug}

Der Chefarzt wurde darüber hinaus noch wegen Betruges verurteilt, weil festgestellt wurde, dass er in mehreren Fällen angeblich von ihm persönlich erbrachte Operationsleistungen gegenüber Patienten hat abrechnen lassen, obwohl er zum Zeitpunkt der Operation nicht im Klinikum war. Dies wurde vom Landgericht als (Abrechnungs-)Betrug gewertet.

Hierbei handelte es sich allesamt um Wahlleistungspatienten. Beispielhaft ist ein Fall, wonach die Operation wie folgt stattfand: Als Operateur begann ein Ober- arzt. Der Angeklagte kam später hinzu. Er verließ den Operationssaal bereits vor Ende der Operation. Es wurde ihm nachgewiesen, dass er vom Fahrdienst des Universitätsklinikums zum Flughafen gefahren wurde, von wo er zu einer Tagung abflog. Während der Autofahrt rief der Angeklagte den Ehemann der operierten Patientin an und erklärte ihm, dass die Operation gut verlaufen sei. Tatsächlich war die Operation zu diesem Zeitpunkt noch nicht beendet, wenngleich der Kern des Eingriffs, die Tumorentfernung, erledigt war. Es waren noch Drainagen zu legen, die Blutstillung vorzunehmen und der Bauchraum zu verschließen. Dies teilte der Angeklagte dem Angehörigen der Patientin jedoch nicht mit. Das tatsächliche Ende der Operation lag etwa eine Stunde nach diesem Anruf. Dennoch rechnete der Angeklagte die ärztlichen Leistungen so ab, als hätte er sie persönlich erbracht. Das Gericht war der Auffassung, dass die in der Liquidation gegebenen Informationen objektiv falsch gewesen seien, denn der Angeklagte hätte als Wahlarzt die Operation nicht (vollständig) persönlich erbracht. Ein Fall einer zulässigen Delegation der Operation auf den Oberarzt gemäß §4 Abs. 2 S. 1 GOÄ hätte nicht vorgelegen. Die Operation als Kernleistung des Chirurgen war nach Auffassung der Richter nicht delegierbar. Selbst wenn man eine Delegation für routinemäßige Leistungen in der Endphase einer Operation für zulässig erachtete, setzte dies zumindest die Möglichkeit des jederzeitigen Eingreifens des Chefarztes voraus, die der ortsabwesende Angeklagte jedoch nicht hatte. Die Leistungen seien auch nicht vom Vertreter persönlich erbracht worden. Die Operation wurde nämlich durch einen Oberarzt ausgeführt, der nicht im Verzeichnis der ständigen Vertreter der liquidationsberechtigten Ärzte ausgewiesen war. Darüber hinaus hätte sich die Vertretungsregelung im Rahmen des wahlärztlichen Vertrages nur auf die unvorhersehbare Abwesenheit des Angeklagten beschränkt, während die reisebedingte Abwesenheit vorhersehbar gewesen sei. Eine mögliche anderweitige Abrede (Individualvereinbarung) mit der Patientin hatte der Angeklagte nicht getroffen.

Dem Angeklagten sei auch bewusst gewesen, dass er vor dem Ende der Operation das Klinikum verlassen hatte und daher zur Abrechnung der Operation als persönliche wahlärztliche Leistung nicht berechtigt gewesen war. Durch die Rechnung wurde beim Empfänger die Fehlvorstellung hervorgerufen, dass der Angeklagte die Operation selbst zu Ende geführt habe.

\section{Steuerhinterziehung}

$\mathrm{Zu}$ guter Letzt wurde der Chefarzt noch wegen Steuerhinterziehung verurteilt, weil er im Rahmen seiner Nebentätigkeit genehmigten Behandlung von Wahlleistungspatienten Einnahmen (u.a. Zahlungen von Patienten ohne Rechnung) erhielt, die er weder gegenüber der Universitätsverwaltung noch in seiner Einkommenssteuer angab. Dadurch wurde sowohl das vom Chefarzt geschuldete Entgelt für die Nutzung der Universitätseinrichtungen als auch die von ihm selbst zu zahlende Einkommenssteuer zu niedrig angesetzt. Die vom Chefarzt eingelegte Revision wurde vom BGH als offensichtlich unbegründet verworfen. Die Verurteilung ist damit rechtskräftig.

\section{Fazit}

Neben der Straftat der Bestechlichkeit bestätigt der BGH mit der Entscheidung nunmehr, dass eine nicht korrekte Abrechnung persönlicher Leistungserbringung auch gegenüber Privatpatienten einen strafbaren Betrug darstellt. Wichtig ist, dass sich Ärzte mit Wahlleistungsberechtigung über die Delegierbarkeit bzw. insbesondere die Nichtdelegierbarkeit bestimmter Leistungen im Klaren sind. Grundsätzlich muss beachtet werden, dass nichtdelegierbare Leistungen persönlich erbracht werden. Delegierbare Leistungen müssen zumindest persönlich beaufsichtigt werden. Gegebenenfalls kann eine zulässige Vertretung, sofern vertraglich abgedeckt, erfolgen. Dies muss aber für den Patienten bzw. Rechnungsempfänger unmissverständlich vor Erbringung der Leistung klar sein. Der Bereich der delegierbaren Leistungen ist seit jeher massiv umstritten. Im Zweifel gilt: Selbst erbringen oder nicht abrechnen.

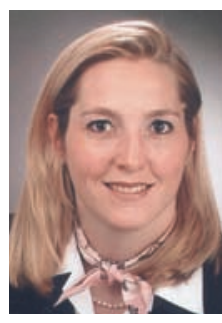

Korrespondenz Dr. iur. Isabel Häser Rechtsanwältin Ehlers, Ehlers und Partner Widenmayerstr. 29 80538 München

Der Beitrag erschien zuerst in der Zeitschrift klinikarzt 11/2011. 